\title{
Ripple Effect: Communicating Water Quality Data through Sonic Vibrations
}

\author{
Dorsey B. Kaufmann \\ University of Arizona, Tucson, AZ \\ dkaufmann2@email.arizona.edu \\ Kunal Palawat \\ University of Arizona, Tucson, AZ \\ kpalawat@email.arizona.edu
}

\begin{abstract}
Pollution in real time can be incredibly powerful, but is difficult to communicate. Persistent deterioration of land, air, and water are largely invisible to the eye and camera lens. What if water itself could visualize its quality and perform the level of contamination? Ripple Effect is an environmental art installation that reveals water contamination through sonic vibrations and light. Using software technology, water contamination levels are translated into sound waves. The installation consists of speakers that play 'data sound tracks', which vibrate water held in attached trays. Participants see and hear the water vibrate based on contaminant concentrations. This paper describes the concept, data-to-sound process, implementation, and participant evaluation surrounding the installation of Ripple Effect in communities neighboring resource extraction and other industrial activity. While there are many existing artworks that visualize environmental quality, Ripple Effect is novel in its use of local water quality data and interactive technology that allows the primary medium, water, to communicate directly with the participant.
\end{abstract}

\section{CCS CONCEPTS}

- Applied computing-Arts and humanities-Media arts.;

\section{KEYWORDS}

Sound computing, data visualization, environmental art, interactive art, sound art, environment, environmental education, MAX/MSP

\section{ACM Reference Format:}

Dorsey B. Kaufmann, Nima Hamidi, Kunal Palawat, and Monica D. RamirezAndreotta. 2021. Ripple Effect: Communicating Water Quality Data through Sonic Vibrations. In Creativity and Cognition (C\&C'21), fune 22, 23, 2021, Virtual Event, Italy. ACM, New York, NY, USA, 7 pages. https://doi.org/10. $1145 / 3450741.3464947$

\section{VIDEO OF INSTALLATION}

https://vimeo.com/308498685.

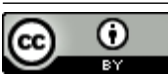

This work is licensed under a Creative Commons Attribution International 4.0 License.

C\&C '21, June 22, 23, 2021, Virtual Event, Italy (c) 2021 Copyright held by the owner/author(s). ACM ISBN 978-1-4503-8376-9/21/06.

https://doi.org/10.1145/3450741.3464947

\author{
Nima Hamidi \\ University of Iowa, Iowa City, IA \\ nima.hamidi.g@gmail.com \\ Monica D. Ramirez-Andreotta \\ University of Arizona, Tucson, AZ \\ mdramire@email.arizona.edu
}

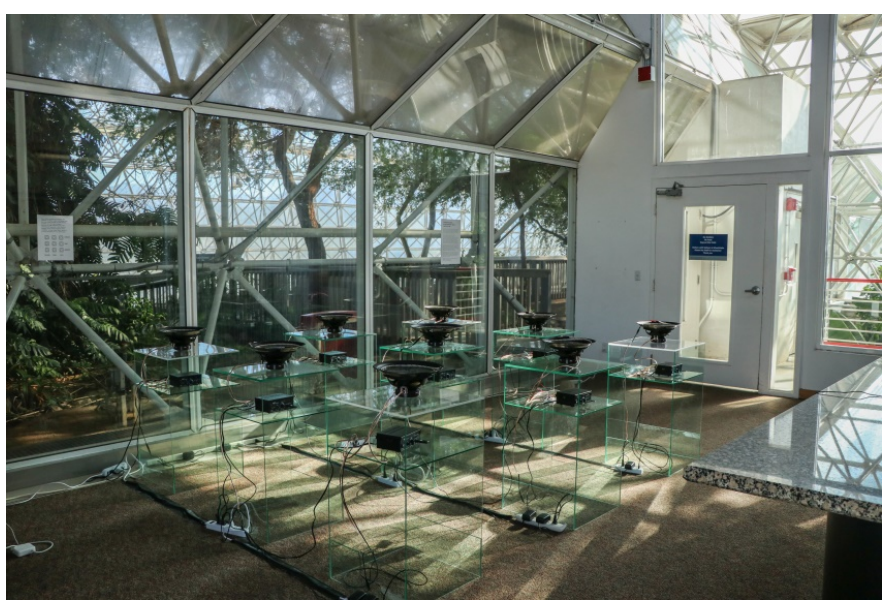

Figure 1: Ripple Effect exhibition at the Biosphere 2, Tucson, AZ, USA.

\section{INTRODUCTION}

Pollution has become the leading global cause of premature death and disease [1]. Effective communication is necessary to create collective environmental action, especially in communities neighboring pollution. Visual art, which uses more emotive and personally relevant language, may help bridge the divide between scientific information and personal and collective action [2].

\section{DESCRIPTION}

Ripple Effect is an interactive art installation that visualizes water quality data and aims to transform how people understand water quality in relation to their environment and how they build connections with natural resources (Figure 1). The installation uses software technology to translate water quality data into sound waves. The installation consists of sound stations that contain speakers with trays of water placed on top. Each station represents a different contaminant that is measured. The speaker plays the soundtrack of the data, meaning participants hear and see the water vibrate based on the chemical concentrations of their community's water. The more active the water, the higher the contaminant concentration. Lining each speaker is an LED light strip, which lights up each time the data points exceed a Maximum Contaminant Level set by a regulatory state or federal agency (e.g. the Environmental Protection Agency's Safe Drinking Water Act) - indicating whether 




Figure 2: Close-up of water vibration and flashing LED lights, South by Southwest festival (SXSW), TX, USA.

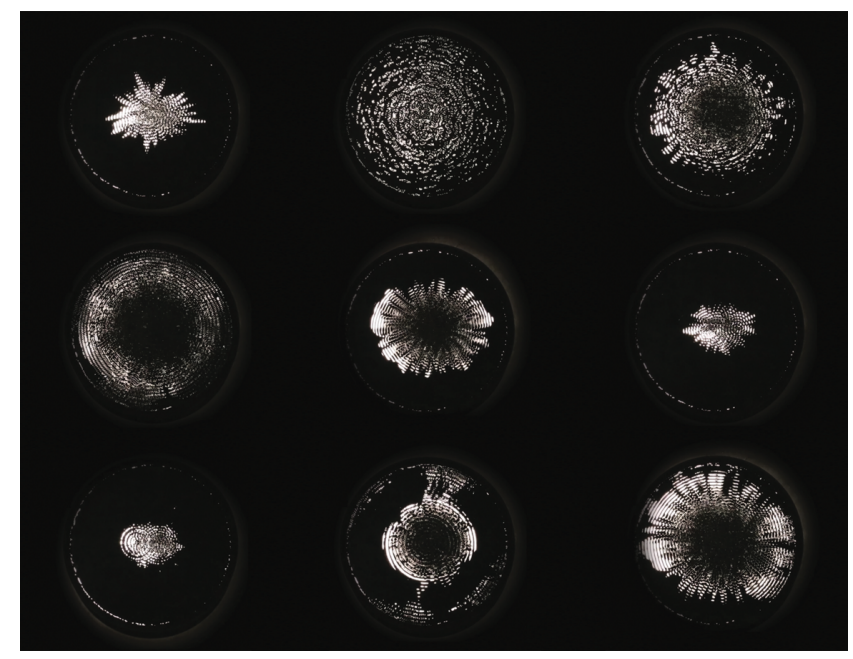

Figure 3: When the sound is played through the water, the vibration creates unique patterns and waves, called water cymatics, as documented in this picture.

or not the water is safe for use (Figure 2). The primary medium in this artwork, water, was selected with the intention of creating a direct connection between the participant to the subject matter as its found in nature. This is what sets the artwork apart from a graph that visualizes water contamination; the primary medium is one and the same as the subject matter. The Max/MSP code acts as a conduit that allows water to communicate its level of contamination to us (Figure 3).

Although researchers have explored visualizing environmental data $[3,4]$ the contribution of contemporary art to the topic is minimal [5]. Ripple Effect grounds the data-driven environmental discussion in a sensorial, empathetic art experience that exists in 3-D space. The data is transformed from rows and columns of numeric values to a living, moving, multi-sensory, 3-D installation that allows the water to directly communicate with participants.

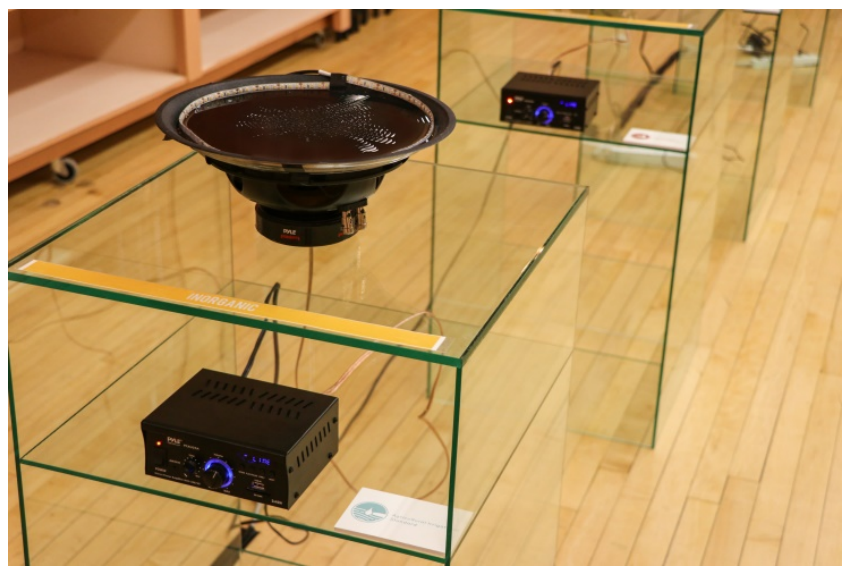

Figure 4: Sound station showing rainwater quality in comparison to an agricultural irrigation standard, Ripple Effect exhibition at the Bullion Plaza Cultural Center and $\mathrm{Mu}$ seum, Globe, AZ, USA.

Ripple Effect serves as a reminder that our actions have a profound and direct impact on the most precious source of life on Earth. Tying to the ripple effect metaphor (ripples expanding across water in response to an initial disturbance), the participants are confronted to reflect on how their and/or society's actions or inactions reverberate throughout the physical and social world.

\section{SET-UP}

Ripple Effect consists of modular sound stations. Each station includes one speaker, one amplifier, a bowl filled with water, a strip of LED lights attached to the rim of the bowl, an Arduino, a label of the contaminant (e.g. Arsenic) and a label of the comparative standard (Figure 4). Across its many exhibitions, the installation has varied from four to nine stations, each representing different contaminants. The installation is accompanied by a legend printed in vinyl on the wall that describes what the sound, ripples, and light mean, along with other details about the exhibition, like what dataset was used. Video and/or large scale prints of the water cymatic patterns have also been shown on the wall behind the sound stations.

\section{DATA-TO-SOUND PROCESS}

The audio is an interactive composition utilizing Max programming language, Max/MSP. This modular software allows the artists to design a set of procedures, or patch, to be processed in real-time. In this work, the Max patch receives the water quality data and uses them as raw material for manipulating the pre-composed audio. The patch consists of many signal-base filters triggered by many thresholds mapped to the contaminant concentrations in the input data. The audio filters alter the frequency, bass, and volume of the output sound. The higher contaminant concentrations are mapped to more intensity or volume of the sound and lower sonic frequencies $(\mathrm{Hz})$, which increases the bass that creates more vibrations and ripples in the water (Figure 5). 


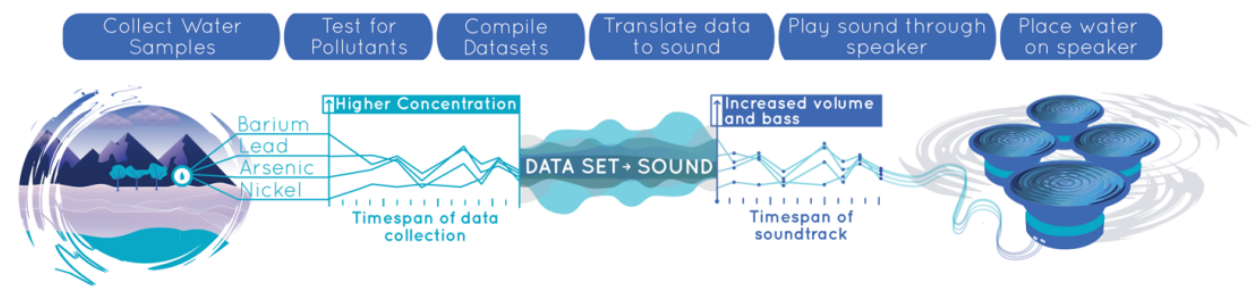

Figure 5: Infographic illustrating Data-to-sound translation.

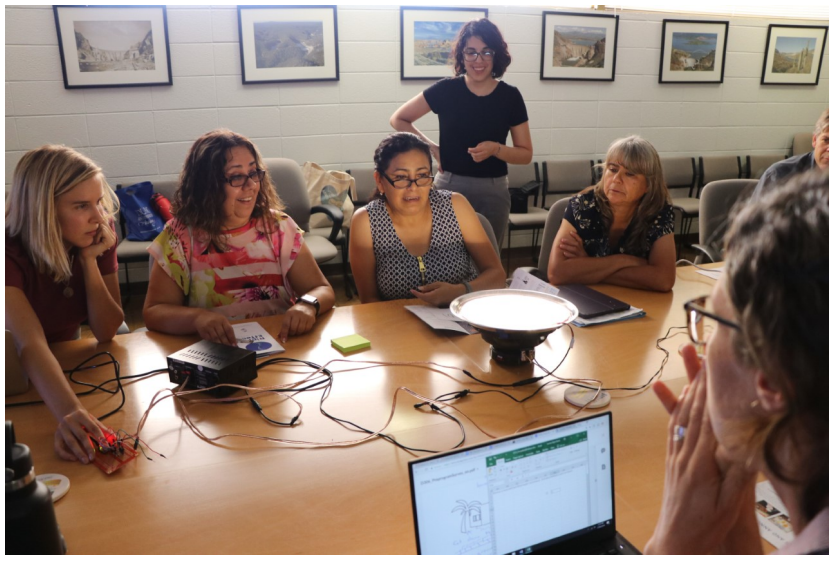

Figure 6: Formative evaluation and beta-testing Ripple Effect with Project Harvest promotoras.

\section{IMPLEMENTATION}

Ripple Effect has communicated water, groundwater, and rainwater data to residents in Arizona, Texas, and Flint, Michigan - which had one of the worst municipal contaminations in history.

The chemical concentrations are derived from either an existing water quality dataset (usually found online or provided by local/state environmental agencies or environmental researchers) or are samples submitted from intrinsically/extrinsically motivated community members for analyses. As a part of the co-created citizen science program Project Harvest, led by the University of Arizona in partnership with Sonora Environmental Research Institute, Inc., researchers and community health workers (promotoras) provided sampling kits to community members throughout Arizona to collect environmental samples on their own property and later, attend community data sharing events with the Ripple Effect installation to see their data come to life [6]. At Project Harvest data sharing events, participants each received a flash drive containing their personalized data to plug into the sound stations.

\section{CROSS-DISCIPLINARY COLLABORATION AND DESIGNING WITH COMMUNITY MEMBERS}

In order to ensure Project Harvest co-produces environmental quality data in a form that will be directly relevant to the participants' lives, increase community involvement in environmental decisionmaking, and improve environmental health literacy $[6,7]$ a series of formative evaluation sessions with promotoras and representative users from each of the targeted Arizona mining communities, as well as other stakeholders and environmental health professionals were held (Figure 6). This formative evaluation and rapid prototyping approach ensures data sharing and visualization methods are informed by the surrounding community's existing perceptions, experiences, attitudes, social background, and cultural orientation, i.e. fulfilling the community's "right to design" [8].

During these initial sessions and throughout development, the Ripple Effect installation was shown to receive feedback on the overall concept, design, and set-up (Figure 6). In these sessions, participants approved Ripple Effect as a way to communicate the rainwater quality results to their respective communities, highlighting that they believed the experience would be engaging. However, due to the level of detail desired, they decided it should not stand alone and results booklet should accompany the exhibition. This booklet complimented Ripple Effect and provided the data in a takehome format that could be shared with family and friends. The artist incorporated participants' feedback into the design of the installation, thus making Ripple Effect a co-created data sharing method that was in collaboration with individuals impacted by environmental pollution.

Working closely with a team of environmental and social scientists also provided an opportunity to maintain the scientific integrity of the data in this exhibition and measure participants' data interpretation, learning outcomes, memory, behaviors, and resulting actions following the exhibition.

\section{PARTICIPANT EVALUATION}

During the exhibitions, participants had some animated reactions to the vibrating water, including open mouths, wide eyes, pointing, leaning down to see the water at eye level, and holding their hands above the water vibrations to catch some of the water droplets.

As a way to measure the impact of this environmental artwork, participants could fill out a survey or join focus groups with moderators after the exhibitions. Focus groups were recorded for further research analysis by a team of learning and social scientists [9].

Many participants shared existing knowledge about environmental contamination, yet reported that the exhibition added a new perspective that reshaped and deepened their environmental understanding. Participants frequently discussed the environmental history of their area and historical sources of pollution together. 


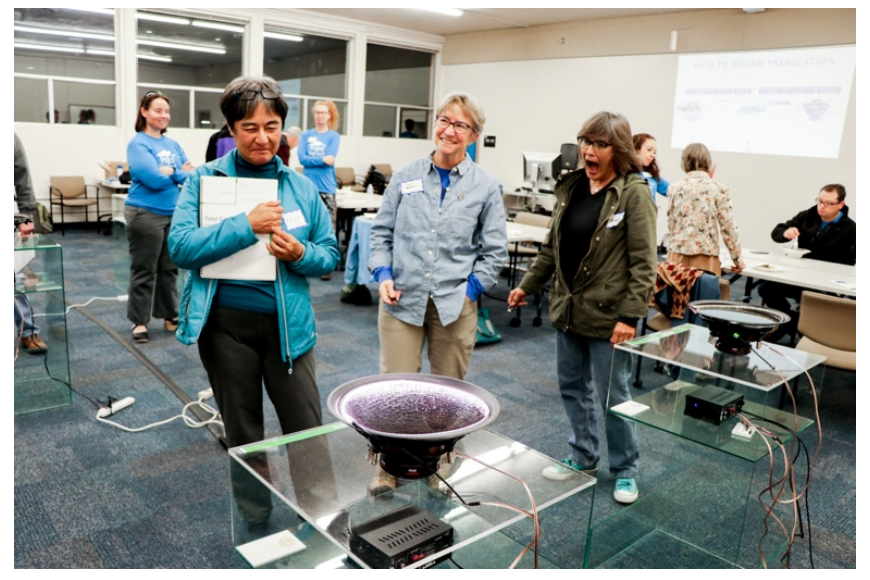

Figure 7: Participants react to the vibrating water, University of Arizona, Tucson, AZ, USA.

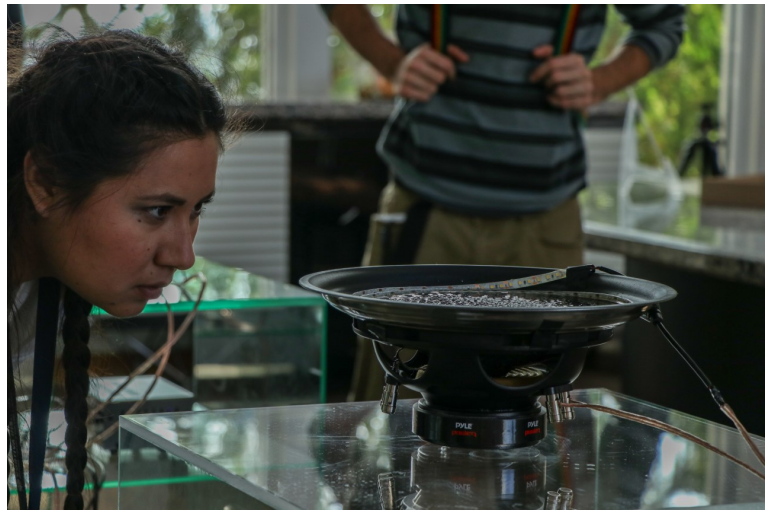

Figure 8: Participant intimately engaging the installation at eye-level to view the vibrating water, Biosphere 2, Tucson, AZ, USA.

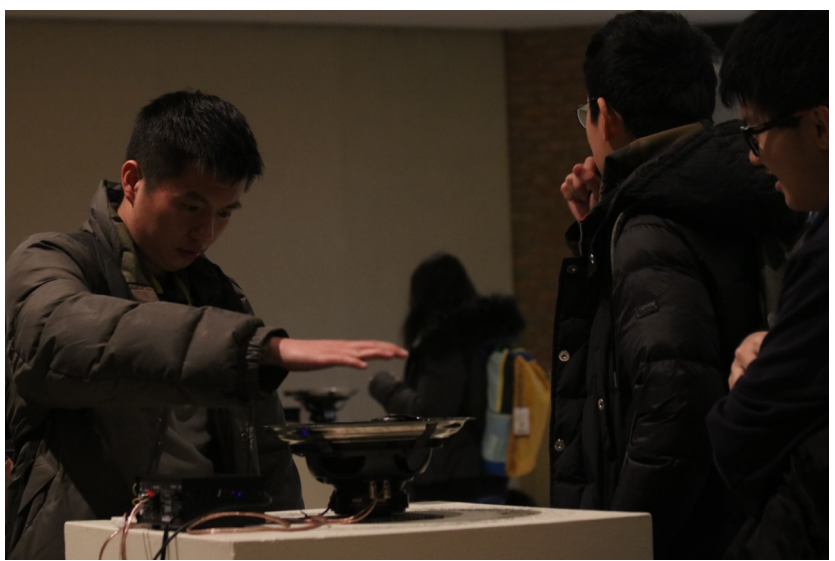

Figure 9: Participant holds out hand above the water tray, Duderstadt Center, University of Michigan, Ann Arbor, Michigan, USA.
When describing Ripple Effect, the participants used the words "suspenseful", "fascinating", "creative", "educational”, "awesome", "multisensory", and "impressive." Fourty-one percent of participants mentioned that Ripple Effect taps into visual/spatial understanding, which benefited their learning experience by affecting their senses. One participant stated 'I get it many ways, because I'm a visual learner ...there is sound, it has light and it also has the vibrations to feel. In my case, it affected all of my senses. So, I loved that." Another participant shared "I think it's a great platform to take a $2 D$ object, graph, and make it a 3D, more visible representation of your data. It just gives you one more way to look at it." One participant emphasized "It's good because you have the visual and you have kind of like a multisensory sort of touch, hearing, vision as well." One participant with poor eyesight preferred listening to the data through Ripple Effect, explaining "for me it's easier to hear it than to see." Twenty-four percent of participants mentioned that Ripple Effect had a greater impact than viewing the same data in a graph. As one participant described "Well I was quite impressed to be able to see the sound samples. In a book, you just see a little dot there and here, you can see it ... 3D or 4D. . and so, it's more exciting." Another participant added "If we saw everything on paper, we would be falling asleep by now-boring! So this is more for us to be able to understand... and also more interesting." One participant also voiced "I think the visual concept really helped, because you can understand the numbers, but when you actually see it and hear it, it makes a bigger impact than just looking at the numbers."

\section{PRELIMINARY STUDY RESULTS}

To determine learning and action outcomes based on data visualization type, the Project Harvest team employed two distinct data sharing experiences and visualizations, Ripple Effect and booklet vs. booklet-only [9]. The study observed that heightened attention and engagement in environmental artworks can have a long-lasting impact, meaning that people are more likely to pay attention and remember their data in the future which can translate into action.

Participants that attended Ripple Effect not only had stronger intentions to act, but were also more likely to follow through with their intentions six months after the data sharing event [9]. Out the participants who gave responses about their intention to take action $(\mathrm{n}=19)$, all of the Ripple Effect participants intended to take action based on their data, whereas less than half of the participants in the booklet-only group reported wanting to change their behavior - most intended not to do anything differently (Figure 11). Based on a chi-squared test, there is a significant relationship between intention to act and data visualization type $(\mathrm{p}<0.05)$. Note that intention to act may be confounded by the individual's rainwater contaminant concentrations.

Ripple Effect participants also recalled the data sharing event or data more specifically than the booklet-only participants, who recalled the event in a broader, less detailed sense (Figure 12) [9]. A chi-squared test showed the relationship between memory recall and data vis type as significant $(\mathrm{p}<0.05)$.

\section{FUTURE DIRECTIONS}

Our results indicate that environmental art can not only communicate complex scientific data effectively, but can also overcome 


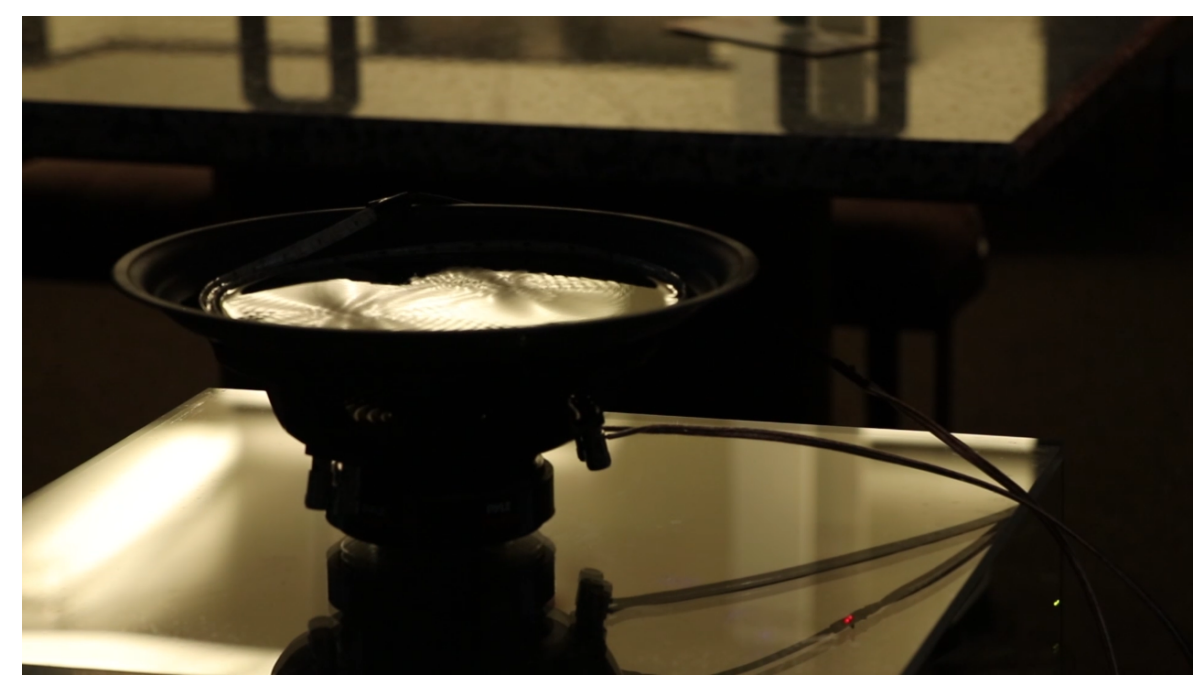

Figure 10: Ripple Effect, South by Southwest festival, Texas, USA.

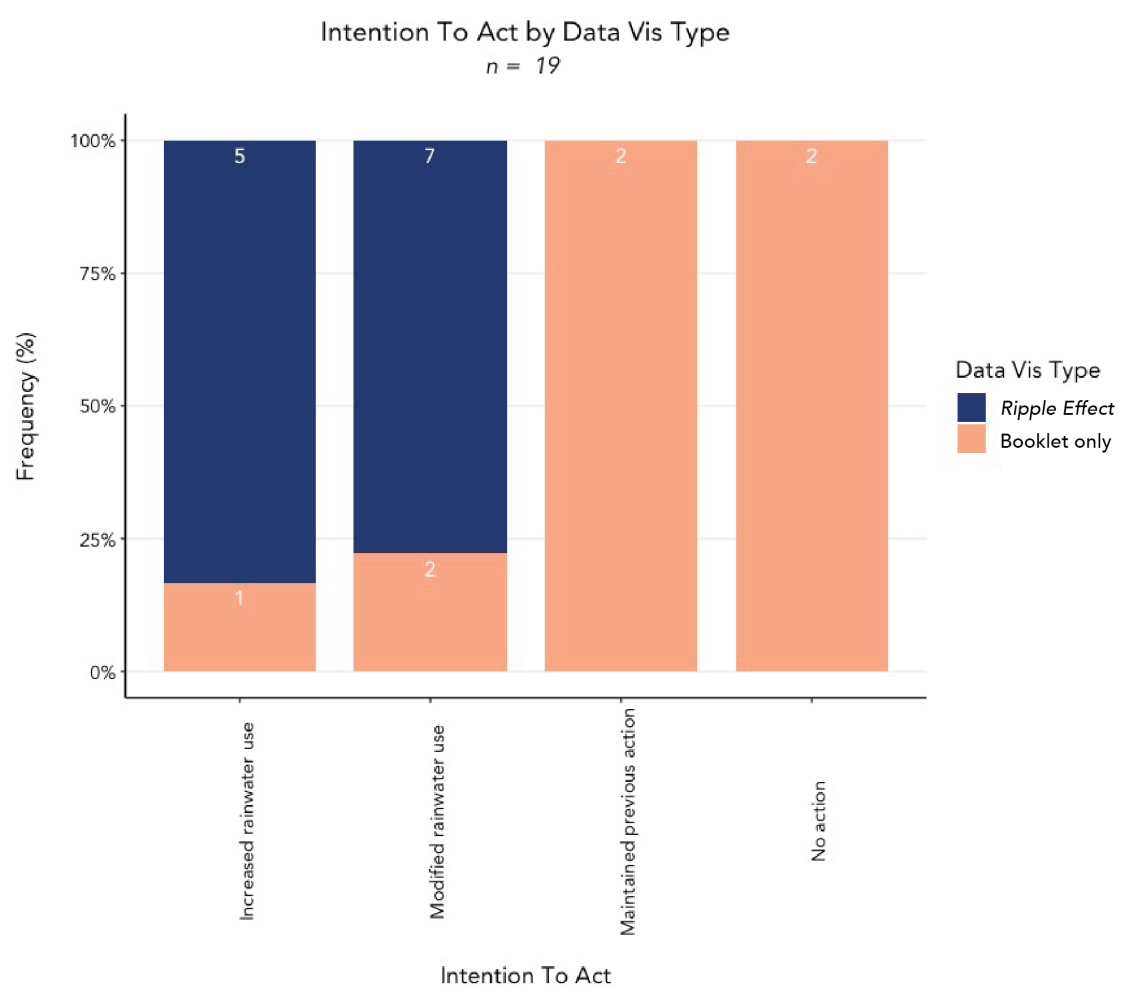

Figure 11: Intention to Act by Data vis type.

knowledge retention and recall, which are known barriers associated with traditional science communication. Ripple Effect participant responses will contribute to the literature in efforts to advance environmental communication and arts research [9]. These findings will identify the aspects of the artwork that are most impactful on how people think and act within their environment, so that environmental art can further be defined, redefined, and implemented in response to the everchanging social, political, and ecological climate. Knowing when, for example, an environmental art approach will be more effective, beneficial, and impactful than a traditional scientific 


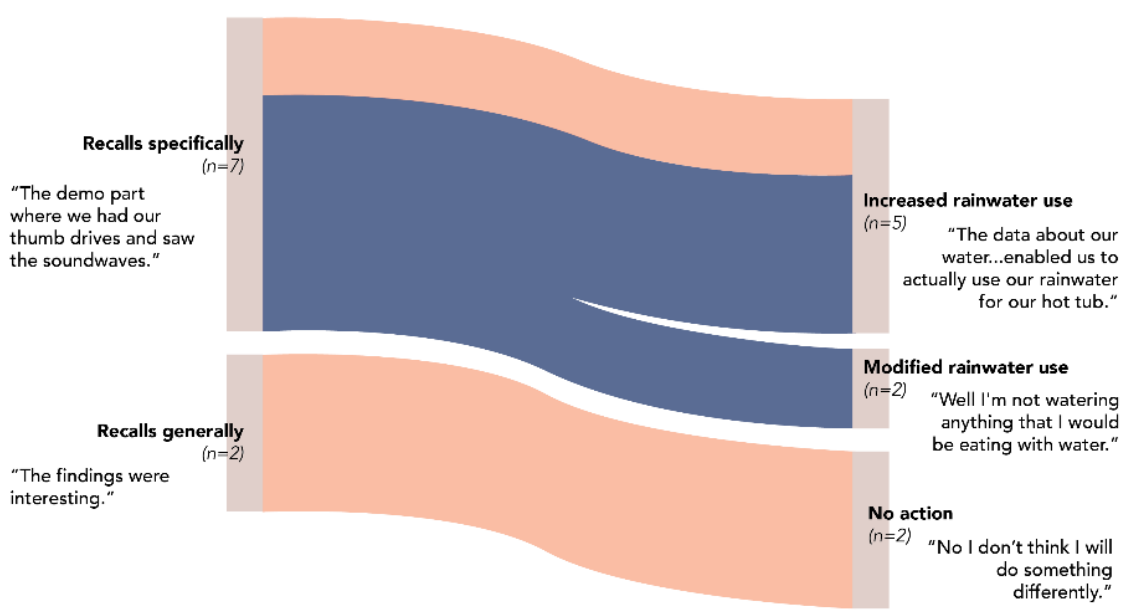

\section{Legend}

Booklet only participants $(n=3)$

Ripple Effect participants $(n=6)$

width of line represents number of responses out of total responses by data-vis type

Figure 12: Sankey diagram showing participant memory recall to action taken by participants.

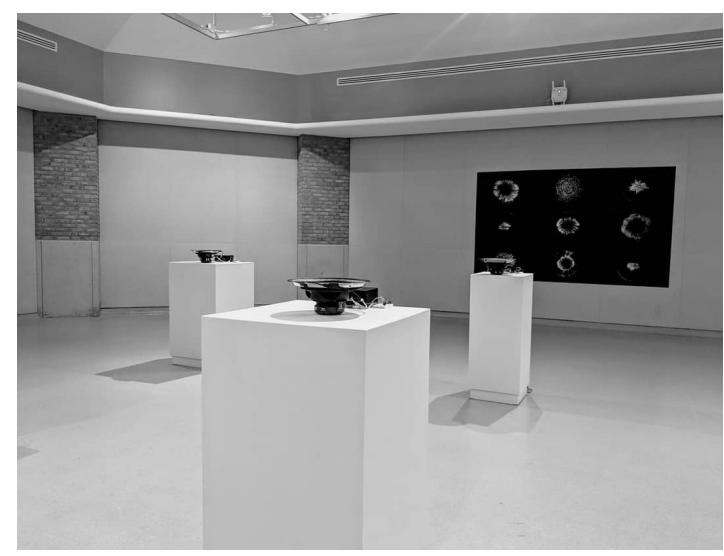

Figure 13: Ripple Effect installation with large scale prints, Duderstadt Center, University of Michigan.

approach will aid researchers, designers, artists, and scientists in their environmental communication efforts.

\section{ACKNOWLEDGMENTS}

We would like to thank Nevan Madrid and Addison Kaufmann for their contribution to Ripple Effect. We also want to thank Shana Sandhaus, Leona Davis, Ariane Mohr-Felsen and Sanlyn Buxner

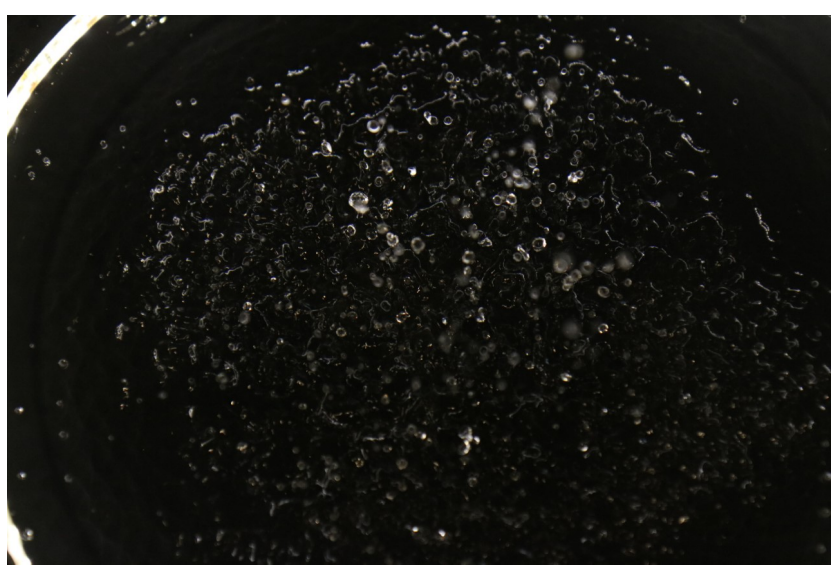

Figure 14: Ripple Effect, view looking down into the tray of vibrating water

for their assistance in the Project Harvest evaluation. Funding for exhibition materials were provided by the Alliance for Art in Research Universities (a2ru) and Biosphere 2. The Project Harvest research and installation materials were funded by the National Science Foundation's Division of Research and Learning grant award number 1612554 . 


\section{REFERENCES}

[1] Landrigan, P. J., Fuller, R., Acosta, N. J. R., Adeyi, O., Arnold, R., Basu, N. (Nil), Baldé, A. B., Bertollini, R., Bose-O’Reilly, S., Boufford, J. I., Breysse, P. N., Chiles, T., Mahidol, C., Coll-Seck, A. M., Cropper, M. L., Fobil, J., Fuster, V., Greenstone M., Haines, A., Zhong, M. (2018). The Lancet Commission on pollution and health. The Lancet, 391(10119), 462-512.

[2] Liselotte Roosen, Christian A. Klockner, and Janet Swim. 2017. Visual art as a way to communicate climate change: a psychological perspective on climate changerelated art. World Art.

[3] Sophie A. Nicholson-Cole. 2005. Representing climate change futures: a critique on the use of images for visual communication. Computers, Environment and Urban Systems.

[4] Stephen R.J. Sheppard. 2012. Visualizing climate change: A guide to visual communication of climate change and developing local solutions. Routledge, New York City, NY.
[5] Laura K. Sommer, Janet K. Swim, Anna Keller, \& Christian Andreas Klöckner. 2019. Pollution Pods: The merging of art and psychology to engage the public in climate change. Global Environmental Change, 59, 101992.

[6] Ramirez-Andreotta MD, Abrell L, Kilungo A, McLain JET, Root R. 2019. Partnering for Action: Community monitoring of harvested rainwater in underserved, rural, and urban Arizona communities. Water Resources IMPACT. 21(2) 12-15.

[7] Davis LF, Ramírez-Andreotta MD, Buxner S. 2020. Engaging Diverse Citizen Scientists for Environmental Health: Recommendations from Participants and Promotoras. Citizen Science: Theory and Practice, 5(1): 7, pp. 1-27. DOI: https: //doi.org/10.5334/cstp.253.

[8] Kaufmann and Ramírez-Andreotta, 2021. "Equity-Centered Community Design: Translating Environmental Data to Support Action", in preparation for Applied Environmental Education \& Communication.

[9] Kaufmann, Palawat, Ramírez-Andreotta, et al. 2021. "Communicating Environmental Data through Art: the role that sentiment and memory play in evoking environmental action", in preparation for Global Environmental Change. 